\title{
UV-spektrometrische Gift-Schnellbestimmung an Mageninhaltsproben unter besonderer Berücksichtigung relativ seltener organischer Vergiftungsmittel
}

\author{
Von D. Tress \\ Aus der Abteilung für Toxikologische Chemie des Instituts fïr Gerichtliche Medizin der Universität Rostock
}

(Direktor: Prof. Dr. W. Haferland)

(Eingegangen am 3. Mai 1969)

\begin{abstract}
Es wird erstmals zusammenhängend über Möglichkeiten der direkten UV-spektrometrischen Bestimmung von Vertretern der großßen Gruppe UV-absorbierender, toxisch-rclevanter organischer Arzneimittel und Gifte an frischen und gelagerten vitalen und mortalen Mageninhaltsproben unterschiedlichster Beschaffenheit berichtet. Die Verfahrensweise, die Voraussetzungen für ihre Anwendung sowie für die Bewertung der Absorptionskurven werden beschrieben und anhand 13 oraler Vergiftungen mit Vertretern verschiedener Wirkstoffgruppen auch bildlich erläutert.

Die Verfahrensweise eignet sich bei oralen akuten Vergiftungen zur schnellen Uberprüfung von Patientenangaben und Ermittlungsergebnissen sowic zur Orientierungsanalyse oder auch als unabhängige Nachweis- und gleichzcitig semiquantitative Bestimmungsmethode für alle klinisch-chemischen und forensisch-toxikologischen Laboratorien, die mit einem leistungsfähigen UV-Spektrophotometer ausgerüstct sind.
\end{abstract}

The rapid UV-spectrophotometric determination of toxic materials in samples of stomach contents, with special reference to the relatively less common organic poisons

The use of UV-spectrophotometry for the determination of the large group of UV-active poisons and potentially toxic pharmaceuticals was studied. The samples of stomach contents studied contained no chemical additives, they were fresh or stored, taken post mortem or from live persons, and were of widely varying composition and condition. The procedure, the conditions for its application and for the evaluation of the absorption curves are described. It is illustrated with 13 different examples of oral poisoning, mostly by the less common toxic agents.

In acute oral poisoning, the method can be used to quickly check information offered by the patient or obtained by enquiry, and for orientation analysis. It can also be used as an independent detection and semiquantitative determination method for all clinical and forensic-toxicological laboratories that have a suitable UV-spectrophotometer.

Bei akuten Vergiftungen kann eine schnelle Ausmittlung und Bestimmung der Wirkstoffe zwecks Einleitung einer gezielten Antidot-Therapie von entscheidender Bedeutung sein. Die klinische Diagnose allein darf in solchen Fällen nur dann als gesichert gelten, wenn die aufgenommenen Wirkstoffe bekannt sind und die Symptome hierdurch ihre Erklärung finden. Bei Suizid- und Mordversuchen sind sichere Kenntnisse über Art und Menge der applizierten Wirkstoffe mit ausreichender Schnelligkeit häufig nur durch chemisch-analytische Untersuchungen zu erlangen.

Dank der ständigen Entwicklung neuer, einfacher Analysenmethoden mit hoher Empfindlichkeit und Spezifität sowie zweckmäßiger Kombinationen dieser Methoden existieren für die häufigsten exogenen Vergiftungsmittel wie Barbitursäurederivate, andere Sedativa, Kohlenmonoxid und Áthylalkohol bereits mehrere unkomplizierte Untersuchungsverfahren (z. B. nach DuNLOP und CurNow (1)), die im Prinzip jedes klinischchemische und forensisch-toxikologische Laboratorium in die Lage versetzen, innerhalb von ein bis zwei Stdn. die Ausmittlung und die quantitative oder semiquantitative Bestimmung dieser und einiger weiterer Wirkstoffe durchzuführen. Als Untersuchungsmaterial werden im klinischen Bereich im allgemeinen Urin-, Blut- und Mageninhalts-Proben, ggf. auch Tatortspuren verwendet.

Die für biologische Materialien beschriebenen systematischen Analysenverfahren setzen durchweg eine
Eliminierung der Wirkstoffe voraus und sind zudem in der Regel mit einer vorausgehenden Eiweißfällung oder/und mit einer folgenden chemischen oder chromatographischen Reinigung und Trennung der extrahierten Substanzen verbunden.

Die Möglichkeiten zur schnellen Gifterkennung an Mageninhalts-Proben werden allgemein zu wenig beachtet, obwohl solche Proben in den meisten Fällen akuter oraler Vergiftungen spontan durch Erbrechen anfallen oder durch Spülung gewonnen werden können, und die Analytik der eingangs genannten und weiterer Vergiftungsmittel (außer Kohlenmonoxid) an derartigen Proben mindestens so schnell und sicher durchführbar ist wie am Blut oder Urin. Dies trifft insbesondere dann $\mathrm{zu}$, wenn die Mageninhalts-Proben in den Gift-Resorptionsphasen gewonnen wurden, die sich bei oraler Applikation hoher Dosen über verhältnismäßig lange Zeit erstrecken können. Auch nach Beendigung der Hauptresorption liegen die Wirkstoffspiegel im Mageninhalt vielfach noch in der Größenordnung der Blutwerte.

Zweck dieser Mitteilung ist es, auf eine von den üblichen toxikologisch-analytischen Methoden weitgehend unabhängige, in der Literatur bisher kaum beachtete Verfahrensweise aufmerksam zu machen, welche die schnelle Erkennung der großen $\mathrm{Zahl}$ wenig flüchtiger organischer Arznei- und Giftstoffe und ggf. gleichzeitig deren semiquantitative Bestimmung ermöglicht: die UV-spektrometrische Untersuchung chemisch unveränderter $\mathrm{Ma}$ geninhalts-Proben oder Extrakte derselben in wäßr. 
Lösung. Hierzu sollen alle Mageninhalt enthaltende Proben zählen, unabhängig von der Art ihrer Gewinnung und Zusammensetzung.

Die Analysenmethodik beruht auf den experimentell gewonnenen Erkenntnissen, daß erstens MageninhaltsProben in der Norm - weitgehend unabhängig von ihrer Zusammensetzung und Beschaffenheit - einen qualitativ gleichartigen Absorptionsverlauf im UVBereich aufweisen und zweitens etwa $90 \%$ aller toxikologisch-bedeutsamen, wenig flüchtigen organischen Wirkstoffe in neutralen und schwach sauren wäßr. Medien ausreichend löslich sind, eine relativ starke UVAbsorption und in beiden Medien ein ähnliches oder identisches Spektralverhalten zeigen.

Die Bedeutung dieser Verfahrensweise zur schnellen Überprüfung von Patientenangaben oder Ermittlungsergebnissen sowie als Vorprobe oder Ergänzung der üblichen Methoden soll anhand von Vergiftungsfällen mit Vertretern 13 verschiedener Substanzgruppen aus unserer toxikologisch-analytischen Routinetätigkeit erläutert werden.

Die gewählten Beispiele beinhalten UV-spektrometrische Analysen an vitalen und mortalen Mageninhalts-Proben unterschiedlichster Beschaffenheit. In allen Fällen wurden die UV-spektrometrischen Befunde sowohl an den Mageninhalts-Proben als auch an weiteren Materialien (Blut, Urin, Organe) durch andere Verfahren zusätzlich gesichert. Diese Untersuchungen sind aus Gründen der Übersichtlichkeit der Abbildungen und des Umfanges der Mitteilung allgemein nicht vermerkt. Angaben zur Vorgeschichte der Vergiftungsfälle werden nur so weit berücksichtigt, wie sie zum Verständnis der anstehenden chemisch-analytischen Probleme notwendig erscheinen.

In mehreren Fällen sind die Nachuntersuchungsergebnisse an ein Jahr oder länger gelagerten MageninhaltsProben dargestellt, um hierdurch zugleich auf die Beständigkeit der Gifte in solchen Proben unter geeigneten Lagerungsbedingungen hinzuweisen. Untersuchungen an länger chemisch-unkonserviert gelagerten Organmaterialien, die hinsichtlich der Aussagekraft der Analysenergebnisse vor allem in forensischen Fällen von Bedeutung sein können, sind bisher in der toxikologischen Literatur nur vereinzelt beschrieben worden.

\section{Material, Methodik und Geräte}

Zur direkten UV-spektrometrischen Untersuchung sind prinzipiell Mageninhalts-Proben aller Art geeignet. Die Lagerung der Proben erfolgte im Kühlschrank bei $0^{\circ}$. Zwecks Aufnahme der Spektren werden Teile der überstehenden Flüssigphase durch Zentrifugieren oder Filtrieren (ggf. Absorption von Filterinhaltsstoffen beachten!) von Festkörpern und Schwebstoffen getrennt. Ist keine ausreichende Flüssigkeitsmenge gewinnbar (z. B. bei Erbrochenem oder angetrockneten Proben), wird zuvor eine Aufschwemmung in Wasser hergestellt.

Die klare wäßr. Phase überprüft man hinsichtlich ihrer Transmission (bzw. Extinktion) bei $250 \mathrm{~nm}$ gegen Wasser, nötigenfalls ist sie so weit mit Wasser zu.verdünnen, daß die Transmission im günstigen Empfindlichkeitsbereich des Spektralphotometers liegt (T $20-40 \%$, E $0,8-0,4)$. Die Aufnahme der Spektren sollte prinzipiell im gesamten meßbaren UV-Bereich, oberhalb $350 \mathrm{~nm}$ nur im Falle eines Wiederanstieges der Absorption, erfolgen. Zur
Steigerung der Erfassungsquote UV-absorbierender Wirkstoffe mit Absorptionsmaxima um oder über $300 \mathrm{~nm}$ können ab $280-300 \mathrm{~nm}$ geringere Verdünnungśgrade zur Untersuchung gelangen, da die Eigenabsorption der Mageninhalts-Proben im nahen UV-Bereich allgemein wesentlich geringer ist (vgl. hierzu Abb. 1 und 2).

Die in dieser Mitteilung dargestellten Spektren wurden entweder mit dem VSU-I (Zeiss-Jena) oder/und mit dem registrierenden Zweistrahlspektrophotometer SF-8 (OMO-Leningrad) unter Benutzung von 0,5- oder 1-cm-Quarzküvetten aufgenommen. Als Vergleich diente stets das verwendete Verdünnungs- oder Lösungsmittel ${ }^{1}$ ).

Bei nichtregistrierenden Geräten empfiehlt sich die Messung bei offensichtlich kontinuierlichem Absorptionsverlauf in Intervallen von 5 (max. 10) nm, im Bereich von Minima und Maxima im Abstand von $1 \mathrm{~nm}$.

Von Mageninhalts-Ausgangsproben und den Meßlösungen werden die pH-Werte bestimmt. Zur Erhöhung der Informationskapazitāt der UV-Spektralanalytik und zur Erfassung solcher Substanzen, die im wäßr. neutralen Medium keinen ausgeprägten Absorptionsverlauf zeigen, werden die gemessenen Lösungen zusätzlich sowohl nach Ansäuerung als auch Alkalisierung (z. B. zur Erkennung der Barbiturate) entsprechend untersucht. Zugabe der Reagenzien (verd. Schwefelsäure, 10 proz. Natronlauge) und Mischen kann in der Küvette erfolgen.

Sofern aus Mageninhalts-Proben Partikel von Tabletten, Dragees oder anderen suspekten Boden- und Schwimmkörpern mechanisch eliminierbar sind, werden von diesen zu Vergleichs- und Sicherungszwecken entsprechende Lösungen bereitet und ausgemessen. Dies gilt sinngemä $\beta$ auch für sonstige Spurenmaterialien (Trinkgefäße, Medikamente, Behälter u. ä.).

Liegen die UV-absorbierenden Wirkstoffe zur Zeit der Probengewinnung nicht mehr in relativem Überschuß in bezug auf die „natürlichen" UV-absorbierenden Mageninhalts-Stoffe vor - z.B. nach Beendigung der Hauptresorption - , so führt allgemein eine einmalige Schüttelextraktion der Original-Probe (ggf. nach $\mathrm{Ab}$ trennung der Fette) mit Ather zur Anreicherung der Fremdstoffe. Bei Verdacht auf stark basische Substanzen ist die Probe vorher zu alkalisieren. Die Abdampfrückstände werden in Wasser - nötigenfalls nach Anlösen mit etwas Methanol - aufgenommen und wie angegeben weiter behandelt.

\section{Ergebnisse}

\section{Mageninhalts-,Norm'-Spektren}

In den Abbildungen 1 und 2 sind die Spektren einiger ausschließlich wasserverdünnter Mageninhalts-Proben, welche keine oder nur sehr geringe Mengen UVabsorbierender Fremdstoffe enthielten, wiedergegeben. Diese Spektren repräsentieren den Absorptionsverlauf von etwa 150 untersuchten derartigen Proben vitalen oder mortalen Ursprungs unterschiedlichster Beschaffenheit. Hieraus ergibt sich ein ,Norm'-Absorptionsmaximum von $265-268 \mathrm{~nm}$.

Abb. 3 zeigt zum Vergleich, Norm'-Spektren von Magensäften in verschiedenen $\mathrm{pH}-$ Bereichen. Diese unterscheiden sich von den ,Norm'-Mageninhalts-Spektren im wesentlichen nur durch die Verschiebung des Maximums um etwa $10 \mathrm{~nm}$ zum längerwelligen Bereich.

1) Bei Verwendung von salzsauren wäßr. Pepsinlösungen bzw. „Norm“-Mageninhalts-Proben (wasserverdünnt) mit entsprechender Durchlässigkeit im Bereich 265-275 nm als Leerwert-Lösung anstelle von Wasser kann die UV-spektrometrische Nachweisgrenze für Fremdstoffe in Mageninhalts-Proben erheblich herabgesetzt werden. Bei adäquaten "Leerwerten" können somit an Original-Mageninhalts-Proben Spektren tesultieren, die im gesamten Kurvenverlauf mit denjenigen von Reinsubstanzen praktisch identisch sind. 


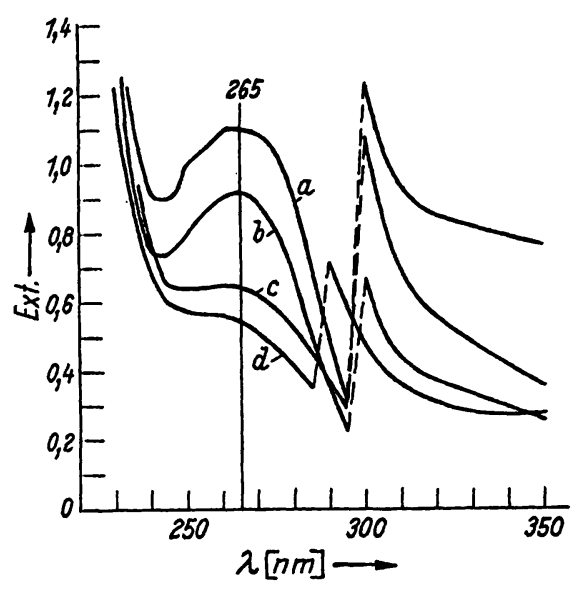

Abb. 1

Mageninhalte, ,Norm'-UV-Spektren, wasserverdünnt gegen Wasser, VSU-I

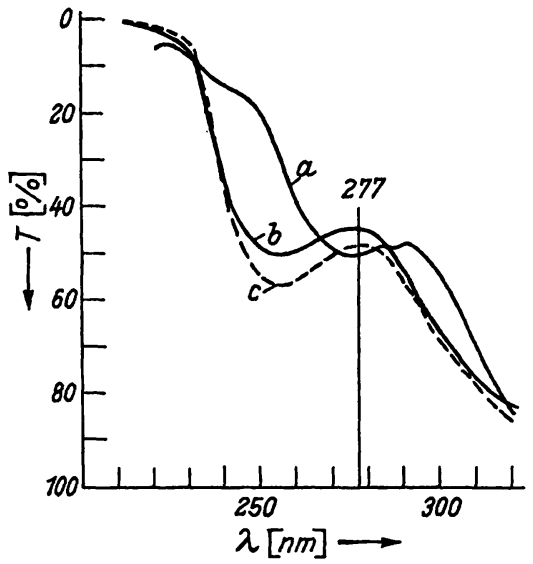

Abb. 3

Magensaft, ,Norm'-UV-Spektren, 1:10 wasserverdünnt gegen Wasser, SF-8, $1 \mathrm{~cm}-$ Küvetten. Repräsentativ für UV-Spektren der Magensäfte von 6 Patienten vor und nach Histamingabe
a) Magensaft, alkalisiert
b) Magensaft, sauer
c) Magensaft, neutralisiert

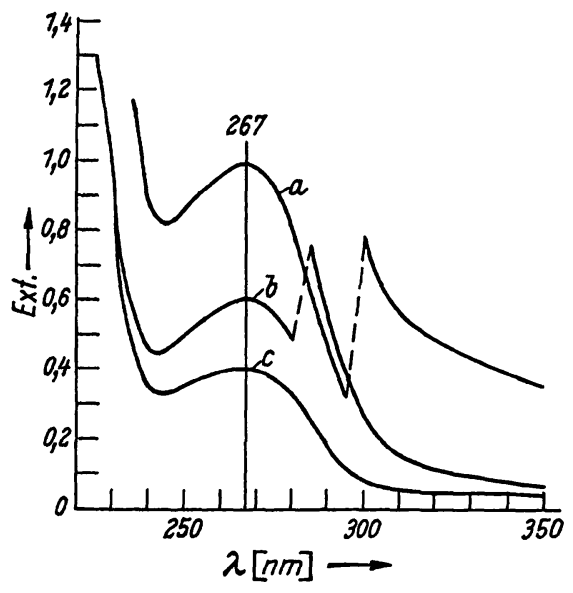

Abb. 2

Mageninhalte, wie Abb. 1. Erklärungen zu den Abb. 1 und 2 siehe Tabelle 1
Original-Mageninhalts-Proben (ausschlicßlich wasserverdünnt)

Der Begriff Original-Mageninhalts-Proben soll in diesem Zusammenhang lediglich zum Ausdruck bringen, $\mathrm{da} ß$ zur Ausmittlung der Fremdstoffe an den übergebenen Mageninhalts-Proben keine chemischen Operationen - ausgenommen eine Wasserverdünnung erforderlich waren.

Benedorm (Persedon, Didropyridinum, 2,4-Dioxo3,3-diäthyltetrahydropyridin)

Vergiftungsverdacht mit unbekannten Mitteln (Tgb.-Nr.: B 27/68)

Zur Untersuchung gelangte ein Teil einer $3 /$ umfassenden Magenspülflüssigkeit.

Abbildung 4 zeigt die UV-Spektren der 1 : 3 mit Wasser verdünnten Magenspülflüssigkeit (a) und einer derartigen Probe nach Alkalisierung (b). Zum Vergleich sind entsprechende Spektren von Tablettenmaterial

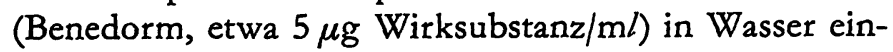
gezeichnet (c, d). Die Spülflüssigkeit enthielt $2,3 \mathrm{mg}$ Didropyridinum $/ 100 \mathrm{ml}$. (1 $\mathrm{cm}$-Küvetten).

Tab. 1

Angaben zu UV-,Norm'-Spektren (Abb. 1 und 2) wasserverdünnter Original-Mageninhalts-Proben

\begin{tabular}{|c|c|c|c|c|c|c|c|c|}
\hline Abb. & $\begin{array}{l}\text { Tox.- } \\
\text { Tgb.-Nr. }\end{array}$ & Alter & $\begin{array}{l}\text { Angegebene } \\
\text { Todes-Ursache }\end{array}$ & $\begin{array}{l}\text { Sektion } \\
\text { p. m. }\end{array}$ & $\begin{array}{l}\text { Mageninhalts- } \\
\text { Beschaffenh. }\end{array}$ & $\begin{array}{l}\text { Wasser- } \\
\text { Verdünnung }\end{array}$ & Küvetten & $\begin{array}{c}\text { Analytische Befunde } \\
\text { am Mageninhalt } \\
\text { (Blut- und Urinbefunde) }\end{array}$ \\
\hline $1 a$ & $152 / 67$ & $25 \mathrm{~J}$. & Verk.-Unfall & 2. Tag & $\begin{array}{l}\text { bräunl. Masse, u.a. } \\
\text { Fleisch, Gurken, } \\
\text { Kartoffeln }\end{array}$ & $\begin{array}{l}1: 100 \text { u. } \\
1: 10\end{array}$ & $1 \mathrm{~cm}$ & $\begin{array}{l}\text { Äthanol } 0,5 \% \\
\text { (Blut } 0,1 \text {, Urin } 0,1 \% \text { ) }\end{array}$ \\
\hline b & $38 / 67$ & $59 \mathrm{~J}$. & $\begin{array}{l}\text { Athanolintox.; } \\
\text { tot aufgefunden }\end{array}$ & 3. $\mathrm{Tag}$ & $\begin{array}{l}\text { aromatisch, über- } \\
\text { wiegend Flüssigkeit }\end{array}$ & $\begin{array}{l}1: 50 u . \\
1: 10\end{array}$ & $1 \mathrm{~cm}$ & $\begin{array}{l}\text { Athanol } 5 \% \text {, Pharmaka } \\
\text { in Spuren (Blut } 3,8 \text {, Urin } \\
4,5 \%, \mathrm{CO}-\mathrm{Hb} 13 \% \text { ) }\end{array}$ \\
\hline c & $62 / 67$ & $62 \mathrm{~J}$. & $\begin{array}{l}\text { Ertrinken (wahr- } \\
\text { scheinl. unmittel- } \\
\text { bar nach } \\
\text { Alkoholgenuß) }\end{array}$ & 1. $\mathrm{Tag}$ & $\begin{array}{l}\text { aromatisch, über- } \\
\text { wiegend Flüssigkeit }\end{array}$ & $1: 5$ & $0,5 \mathrm{~cm}$ & $\begin{array}{l}\text { Athanol } 5 \% \text { (Blut } 0,15 \text {, } \\
\text { Urin } 0,1 \% \text { ) }\end{array}$ \\
\hline d & $118 / 67$ & $40 \mathrm{~J}$. & $\begin{array}{l}\text { Cyanid-Intox. (in } \\
\text { Komb. mit Barbital } \\
\text { u. Athanol) }\end{array}$ & 1. $\mathrm{Tag}$ & $\begin{array}{l}\text { Überwiegend } \\
\text { Flüssigkeit, pH } \\
\text { etwa } 11, \mathrm{HCN}- \\
\text { Geruch }\end{array}$ & $\begin{array}{l}1: 50 \mathrm{u} . \\
1: 20\end{array}$ & $0,5 \mathrm{~cm}$ & $\begin{array}{l}\text { KCN } 1,5 \% \text {, Cyan-Met-Hb, } \\
\text { Äthylcrotylbarbitursäure } \\
3 \text { mg/100 ml, Hämatin } 35 \% \text {, } \\
\text { Åthanol } 5 \% \text {, (Blut } 0,2, \\
\text { Urin } 0,45 \% \text {, }\end{array}$ \\
\hline $2 a$ & $136 / 67$ & $45 \mathrm{~J}$. & $\begin{array}{l}\text { Ertrinken u. } \\
\text { Thorax-Kompr. } \\
\text { (Betriebs-Unfail) }\end{array}$ & 2. Tag & $\begin{array}{l}\text { überwiegend } \\
\text { Flüssigkeit } \\
\text { (Tümpelwasser) } \\
\text { faulig, pH } 5-6\end{array}$ & $\begin{array}{l}1: 15 \text { u. } \\
1: 3\end{array}$ & $0,5 \mathrm{~cm}$ & $\begin{array}{l}\text { Äthanol } 1,4 \% \text { (Blut } 0,4 \text {, } \\
\text { Urin } 0,4 \% \text { oo }\end{array}$ \\
\hline b & $20 / 67$ & $65 \mathrm{~J}$. & $\begin{array}{l}\text { Athanolintox. } \\
\text { bzw. Herztod; } \\
\text { tot aufgefunden }\end{array}$ & 2. Tag & $\begin{array}{l}\text { überwiegend } \\
\text { Flüssigkeit, } \\
\text { pH } 5\end{array}$ & $\begin{array}{l}1: 40 \mathrm{u} . \\
1: 20\end{array}$ & $0,5 \mathrm{~cm}$ & $\begin{array}{l}\text { Äthanol } 5,7 \% \text { (Blut } 2,5 \text {, } \\
\text { Urin } 3,8 \% \text { ) }\end{array}$ \\
\hline c.. & $214 / 67$ & $22 \mathrm{~J}$. & $\begin{array}{l}\text { Athanolintox.; } \\
\text { evt1. Myocarditis }\end{array}$ & 2. $\mathrm{Tag}$ & $\begin{array}{l}\text { überwiegend } \\
\text { Flüssigkeit, } \\
\text { pH etwa 4. }\end{array}$ & $1: 80$ & $0,5 \mathrm{~cm}$ & $\begin{array}{l}\text { Athanol } 1,6 \% \text { (Blut } 4,2 \text {, } \\
\text { Urin } 5,3 \% \text { ) }\end{array}$ \\
\hline
\end{tabular}




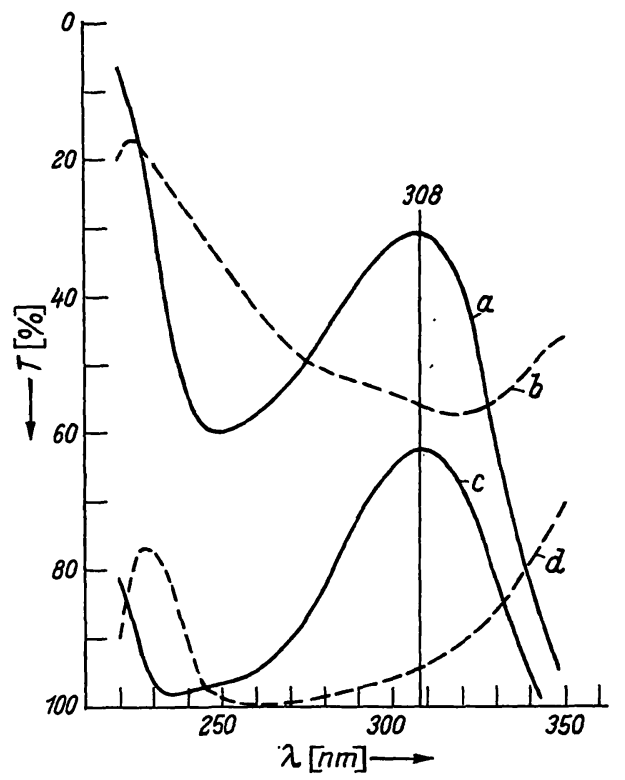

Abb. 4

Benedorm-(Didropyridinum-)Intoxikation, vgl. auch Text

Bi 58 (Dimethoat, O,O-Dimethyl-S-(N-methyl)-carbamylmethyldithiophosphat)

Vollendeter Suizid durcb Trinken des Pflanzenscbädlingsbekämpfungsmittels Bi 58 (Tgb.-Nr.: B 145/65)

Die Sektion erfolgte zwei Tage p. m.

Abbildung 5 gibt die UV-Absorptionskurve einer mit Wasser 1:180 verdünnten Mageninhalts-Probe (a) und zum Vergleich (b) die vom wasserverdünnten OriginalBi 58 (etwa $0,25 \mathrm{mg} / \mathrm{m} /$ ) wieder. (1 cm-Küvetten).

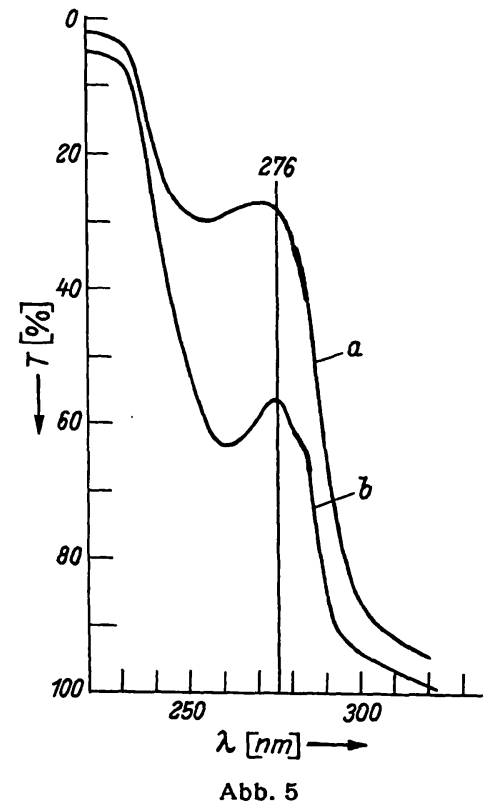

Bi 58-(Dimethoat-)Intoxikation, vgl. auch Text

Die Aufnahme der dargestellten Mageninhalts-Kurve erfölgte nach über zweijähriger Lagerung der Originalprobe. Während der Lagerzeit zeigte sich keine wesentliche Veränderung ihrer chemischen Zusammensetzung und ihres Spektralverhaltens. Der Geruch des Bi 58 war noch in der Mageninhalts-Verdünnung von 1:180 sicher wahrnehmbar. Bei der Bewertung der Absorptionskurve ist insbesondere die rechtsseitig vom Maximum erkennbare Schulter zu beachten, jedoch weist auch der erforderliche Verdünnungsgrad auf das Vorliegen einer UV-aktiven Fremdsubstanz hin. Die über- gebene Mageninhalts-Probe (etwa $200 \mathrm{~g}$ ) enthielt gemäß chromatographisch-chemischer und weiterer UV-spektrometrischer Bestimmungen etwa $50 \mathrm{~g}$ der $\mathrm{Bi}$ 58-Formulierung, die sịch laut Prospekt wie folgt zusammensetzt: $38 \%$. Wirkstoff (Dimethoat), $2 \%$ Emulgator, 60\% Lösungsmittel. Der Wirkstoff besteht aus O,ODimethyl-S-(N-methyl)-carbamylmethyldithiophosphat.

\section{Colchicin}

Suizid-Versuch mit tödlichem Ausgang (Tgb.-Nr.: B 12/67)

Die Giftaufnahme erfolgte 2 Tage vor dem Tode, die Sektion 30 Stdn. p. m. Zur Untersuchung gelangten $200 \mathrm{~g}$ des Leichen-Mageninhalts, der auffallend schmutziggrünlich und kohlehaltig war.

In Abbildung 6 sind die UV-Spektren der 1:60 (a) und 1:180 (c) mit Wasser verdünnten Mageninhalts-Probe (14 Monate p. m.) wiedergegeben und zum Vergleich die UV-Kurven eines alkalischen Mageninhalts-Extraktes (b); dessen Abdampfrückstand in Methanol gelöst wurde, und von Colchicin puriss. in Wasser $(4 \mu \mathrm{g} / \mathrm{m} l)$ (d) dargestellt. (1 cm-Küvetten).

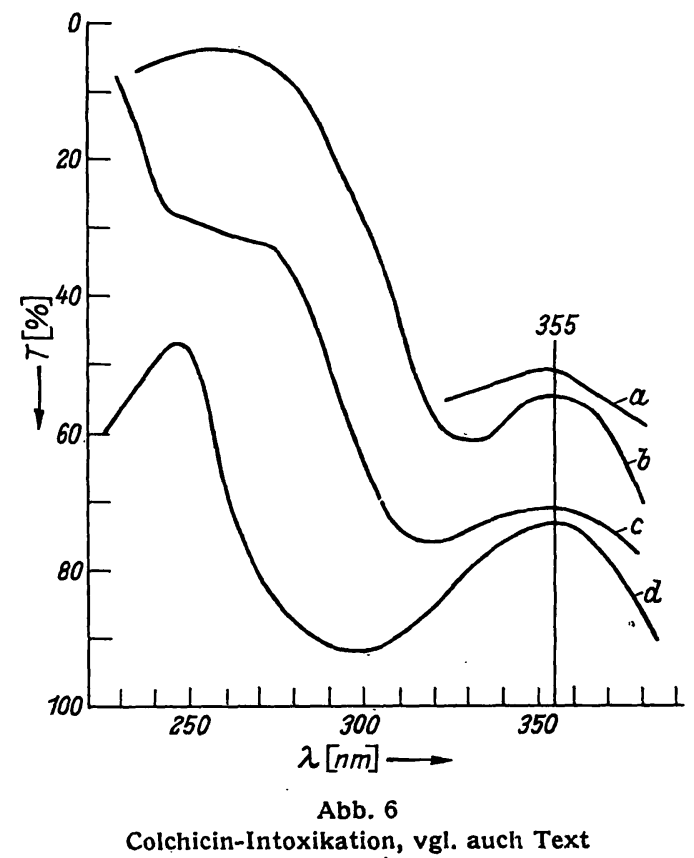

Der Verlauf der Kurve b entspricht dem aus $0,5 \mathrm{ml}$ Mageninhalt extrahierten Colchicin/ml Meßlösung. In der Mageninhalts-Probe befanden sich zur Zeit des Todes noch letale Mengen an chemisch unverändertem Colchicin (neben Colchicein), welches teilweise an Kohle ,gebunden“ war.

INH (Isoniazid, Isonicotinsäurehydrazid)

\section{Akut tödliche suizidale Vergiftung (Tgb.-Nr.: B 99/64)}

Die Leichen-Mageninhalts-Probe bestand aus etwa $500 \mathrm{ml}$ Aktiv-Kohlehaltiger Spülflüssigkeit.

Abbildung 7 gibt die UV-Spektren der 1:60 wasserverdünnten Mageninhalts-Probe (a) und zum. Vergleich diejenigen von Rezeptur-INH $(10 \mu \mathrm{g} / \mathrm{m} l)$ in Wasser (c) und in $0,2 \mathrm{~N} \mathrm{H}_{2} \mathrm{SO}_{4}$ (b) wieder. (0,5 cm-Küvetten).

Die INH-Spektren im neutralen und sauren Bereich unterscheiden sich deutlich. Das Absorptionsmaximum 


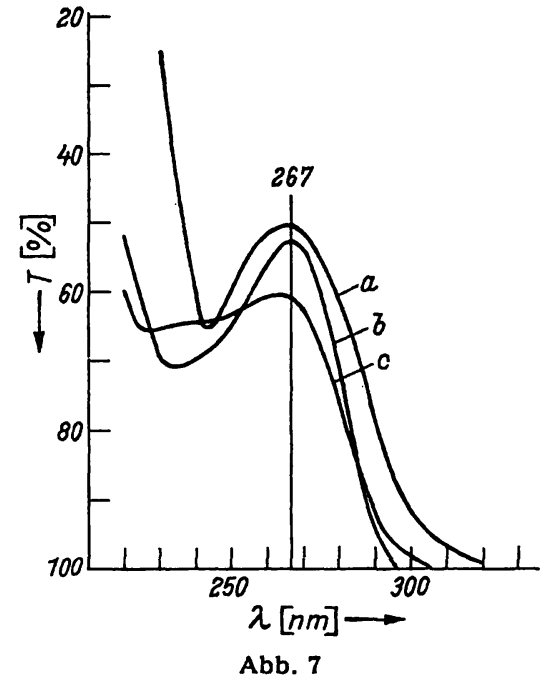

INH-(Isoniazid-)Intoxikation, vgl. auch Text

des Isoniazids in saurer wäßr. Lösung gleicht dem des ,Norm'-Mageninhalts (vgl. Abb. 1 und 2), so daß in diesem Fall zum direkten spektralanalytischen Nachweis Untersuchungen in anderen $\mathrm{pH}-$ Bereichen unbedingt erforderlich waren. Die Mageninhalts-Probe enthielt etwa $1 \mathrm{mg} \mathrm{INH/g}$ Flüssigphase.

Sinophenin (Promazin, 10-(3-Dimethylaminopropyl)phenothiazin)

Vollendeter Suizid durcb Sinophenin, Hexobarbital und Äthanol (Tgb.Nr.: B 71/67)

In Abb. 8 sind die UV-Spektren von 1:90 wasserverdünntem Mageninhalt (a) - hier nach einjähriger Lagerung - und von wäßr. Sinophenin-Lösung (b) (etwa $10 \mu \mathrm{g}$ Wirksubstanz/ml) dargestellt. ( $1 \mathrm{~cm}-\mathrm{Kü}-$ vetten).

Die gestrichelt eingezeichneten Spektren sollen auf die Möglichkeit hinweisen, durch einmalige Atherextraktion der schwach sauren Original-Mageninhalts-Probe - hier ebenfalls nach einjähriger Lagerung - Barbitursäure- und Phenothiazinderivate eliminieren und an der gleichen wäßr. Lösung des Atherabdampfrückstandes UV-spektrometrisch erkennen zu können, wenn Verdünnungsgrad

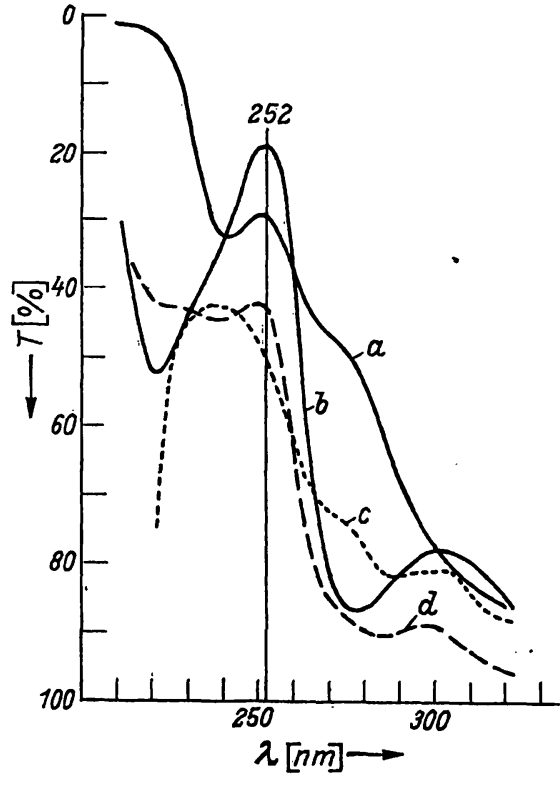

Abb. 8

Sinophenin-(Promazin-)Intoxikation, vgl. auch Text und $\mathrm{pH}$-Wert der Meßlösung entsprechend variiert werden. Das Spektrum d zeigt die Absorption der wäßr. Lösung des Abdampfrückstandes, das Spektrum c die der gleichen Lösung nach dem Alkalisieren. Der Verlauf der Kurven c und d entspricht dem eliminierten Hexobarbital und Promazin aus etwa $0,02 \mathrm{~m} /$ Mageninhalt je $\mathrm{ml}$ Meßlösung.

Summavit (Nicotinsäureamid, Drageefarbstoff)

Vergiftungsverdacht mit unbekannten Mitteln (Tgb.-Nr.: B 11/68)

Ubersandt wurde eine Mageninhalts-Probe von $20 \mathrm{~g}$ (wahrscheinlich Spülfü̈ssigkeit) zur Untersuchung auf ,Barbiturate und andere Tabletten'.

Bei der makroskopischen Betrachtung der Probe fielen der rötlich-gelbe Farbton des Flüssiganteils sowie Tabletten- bzw. Drageebestandteile als Boden- und Schwimmkörper auf. Farblich, geruchlich wie auch chemisch und spektralanalytisch entsprach die Probe einer gesättigten Summavit-Aufschwemmung. Andere übliche Suizid-Pharmaka konnten nicht nachgewiesen werden.

In Abbildung 9, I sind die UV-Absorptionskurven der 1: 144 mit Wasser verdünnten Mageninhalts-Probe (a), einer wäßr. Summavit-Aufschwemmung (c), einer wäßr. alkalisierten Summavit-Aufschwemmung (b) und des am stärksten UV-absorbierenden Bestandteils des Vitaminpräparates, Nikotinsäureamid, in künstlichem Magensaft $(6,7 \mu \mathrm{g} / \mathrm{m} l)$ (d) dargestellt.

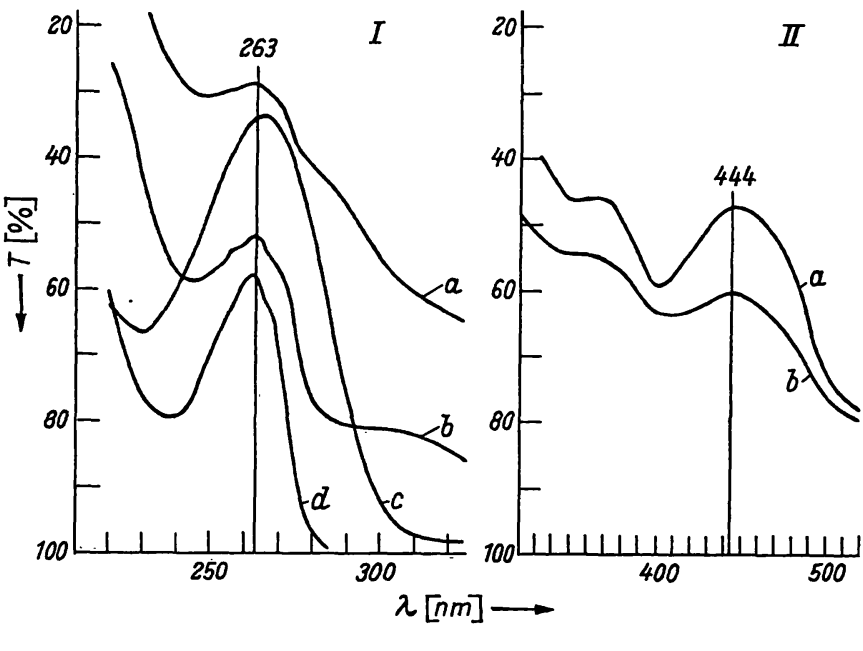

Abb. 9

"Summavit-Intoxikation". I = Nicotinsäureamid, II = Drageefarbstoff, vgl. auch Text

Die VIS-Spektren (II) - a zeigt ein Summavit-Dragee in Wasser angelöst, $\mathrm{b}$ die 1:12 mit Wasser verdünnte Mageninhalts-Probe - können bei farbigem Untersuchungsmaterial, wie in diesem Fall, mit Erfolg zur zusätzlichen Sicherung herangezogen werden. $(1 \mathrm{~cm}$ Küvetten).

\section{Tinctura Aurantii (Pomeranzenöl)}

Akut-tödliche Kombinations-Vergiftung (Cbloralbydrat-Rezeptur, Äthanol, Desinfektionsmittel $C_{4}$, Barbiturate und Analgetika) (Tgb.-Nr.: B 113/64)

Mikroanalytische Untersuchungen an der übergebenen Leichen-Mageninhalts-Probe (etwa $5 \mathrm{~g}$ ) bewiesen das Vorliegen der angegebenen Wirkstoffe. Abbildung 10 


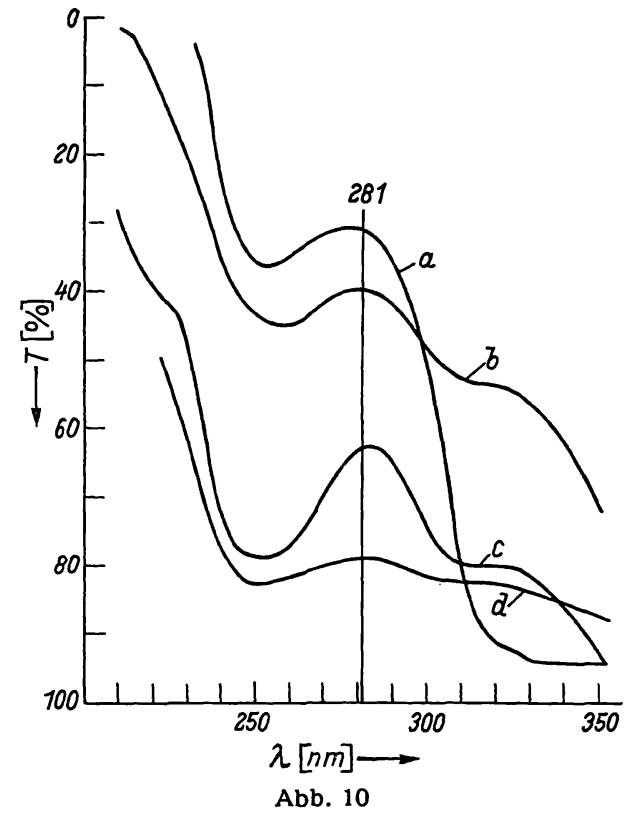

Chloralhydrat-Rezeptur-Intoxikation (Tinctura Aurantii), vgl. auch

zeigt das UV-Spektrum der 1:70 wasserverdünnten Mageninhalts-Probe (b). Zum Vergleich sind die Spektren der wäßr. Spülfüssigkeit der geleerten Tatflasche beschriftet mit ,Chloralhydrat-Rezeptur ${ }^{c}$ - (d) sowie die wäßr. Verdünnung $(1: 9)$ einer frischen Chloralhydrat-Rezeptur (c) und der Tinctura Aurantii $(1: 200)$ (a), der UV-absorbierenden Komponente der Rezeptur, eingezeichnet $(0,5 \mathrm{~cm}-\mathrm{Küvetten})$.

Die übrigen Fremdstoffe der Mageninhalts-Probe, die im sauren oder neutralen wäßr. Medium ebenfalls erfaßbar sind $\left(C_{4}\right.$, Analgetika), störten wegen relativ geringer Konzentration bei dem gewählten Verdünnungsgrad die direkte Bestimmung der Tinctura Aurantii nicht (Näheres siehe unter (2)).

\section{Kalypnon (Äthylcrotylbarbitursäure)}

Suizid mit unbekannten Mitteln (Tgb.-Nr.: M 47/69)

Zur Verfügung stand eine Leichen-Mageninhalts-Probe (graubraune Flüssigkeit, schlammartiger Bodensatz mit weißen Partikeln durchsetzt).

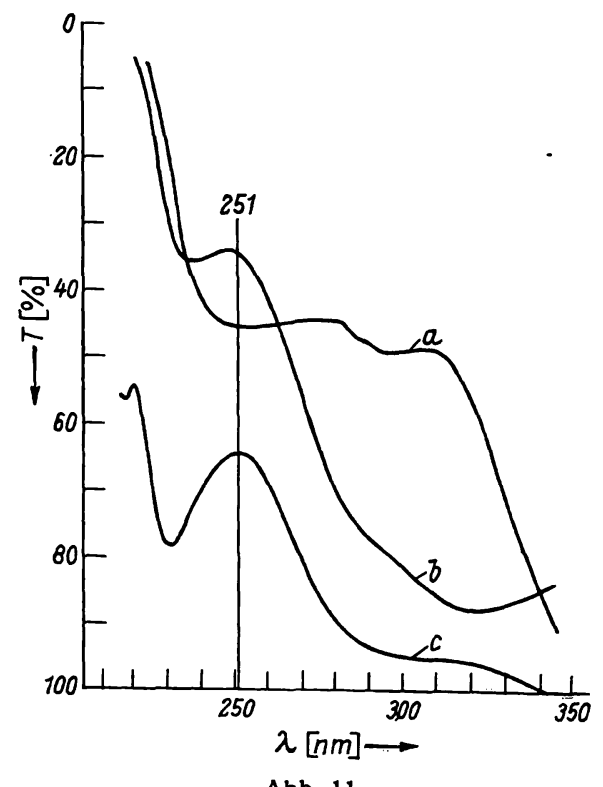

Kalypnon-(Äthyl-crotyl-barbitursäure-)Intoxikation, vgl. auch Text
In Abbildung 11 sind die UV-Spektren einer 1:350 wasserverdünnten Probe des Mageninhalts-Flüssiganteils (a), einer $1: 600$ wasserverdünnten und alkalisierten Probe (b) und zum 'Vergleich Äthylcrotylbarbitursäure in $0,2 \mathrm{~N} \mathrm{NaOH}(7,5 \mu \mathrm{g} / \mathrm{m} /)$ (c) dargestellt.

Das Spektrum a der annähernd neutralen MageninhaltsProbe weicht deutlich von der Norm ab. Die Mageninhalts-Probe enthält demzufolge wahrscheinlich weitere Fremdsubstanzen, die im neutralen und schwach sauren Medium absorbieren. Der Absorptionsverlauf der angesäuerten Probe ist mit a identisch.

\section{Mageninhalts-Proben (extrahiert oder $\mathrm{pH}$-reguliert)}

Bei den folgenden Beispielen handelt es sich ebenfalls um orale Vergiftungen durch Mittel, welche - wie die zuvor angeführten - prinzipiell an wasserverdünnten Original-Mageninhalts-Proben UV-spektrometrisch direkt nachweisbar sind. Mit Ausnahme der Benzalkoniumchlorid- und der Nicotinvergiftung war eine vorherige Anreicherung der Wirkstoffe allgemein deshalb erforderlich, weil zur Zeit der Probenentnahme bzw. des Todes bereits ein Verteilungṣgleichgewicht vorlag oder eine Magenspülung vorangegangen war und die UVAktivität der natürlichen Mageninhalts-Stoffe die direkte Erkennung der Wirkstoffe beeinträchtigte oder verhinderte.

Die Anreicherung erfolgte mịt organischen Lösungsmitteln - allgemein aus chemisch unveränderten Proben -, die spektralanalytische Bestimmung an den wäßr. Lösungen der Abdampfrückstände der Extrakte.

$\mathrm{C}_{4}$ (Benzalkoniumchlorid, n-Alkyl-dimethyl-benzylammoniumchlorid)

Suizidale akut-tödliche Vergiftung durch unverdïnntes Desinfektionsmittel $C_{4}$ (Tgb.-Nr.: B 12/64)

Die Sektion erfolgte am 4. Tag p. $\mathrm{m}$.

Abbildung 12 gibt die UV-Spektren eines aliquioten Teils des eingedampften, in Wasser aufgenommenen Chloroformextraktes der alkalisierten MageninhaltsProbe (b), einer wäßr. Spüllösung des geleerten Trinkgefäßes (a) und eines etwa 1:1000 mit Wasser verdünn-

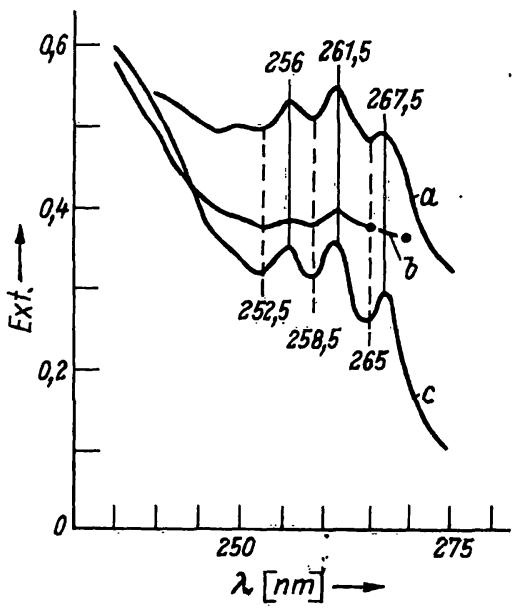

Abb. 12

$\mathrm{C}_{4}$-(Benzalkoniumchlorid-)Intoxikation, vgl. auch Text 


\section{MERCK}

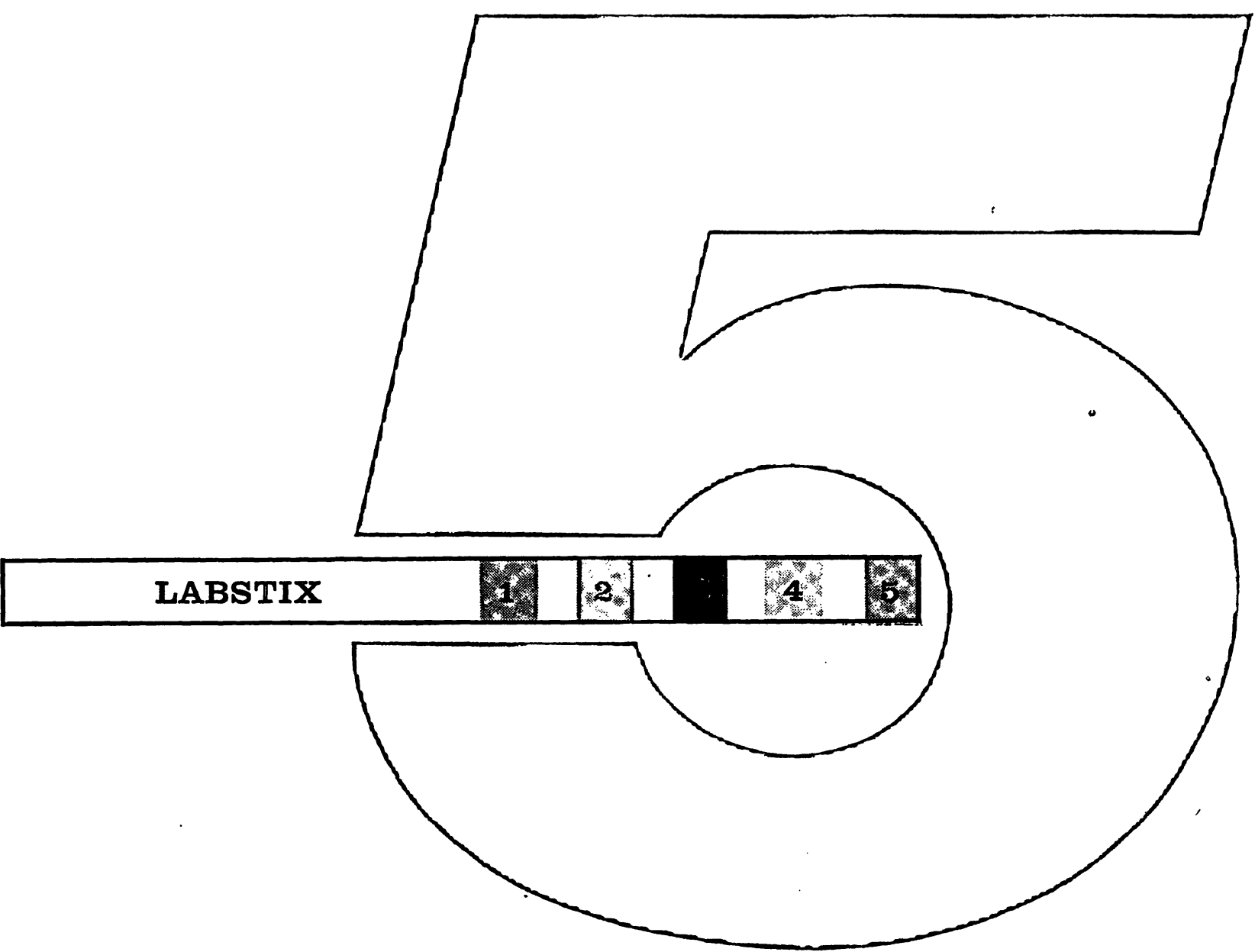

5 wichtige Untersuchungsergebnisse in 30 Sekunden Labstix das Labor in der Westentasche

Der Plastikstreifen mit fünf Reaktionszonen aus imprägnierter Zellulose dient gleichzeitig :

zum spezifischen Nachweis von Glukose zur grobquantitativen Bestimmung von Eiweiß zum Nachweis von Ketonkörpern zum Nachweis von Blut zur Bestimmung des pH-Wertes

Sowohl in der Praxis als auch am Krankenbett ist Labstix ein zuverlässiges Hilfșmittel für die Diagnose.

Verlangen Sie bitte Informationsmaterial.

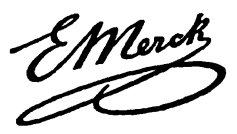


Für schnelle Konzentrierung, Reinigung und Fraktionierung von biologischen Flüssigkeiten

\section{ㅁำ \\ RAEMBRAN ULTRAFILTRATION}

DIAFLO Membranen, in AMICON UItrafiltrationszellen eingesetzt, konzentrieren verdünnte Proteinlösungen innerhalb kurzer Zeit 10 bis 1000-fach. Eine vollständige Beseitigung der Salze oder anderer kleiner Moleküle kann innerhalb der gleichen Zeit mittels Diafiltration erzielt werden.

DIAFLO Membranen verstopfen nicht und sind wieder verwendbar. Jeder Membrantyp besitzt eine bestimmte chemische Beständigkeit und charakteristische physikalische Eigenschaften. Alle Membranen sind nicht denaturierend und biologisch inert.

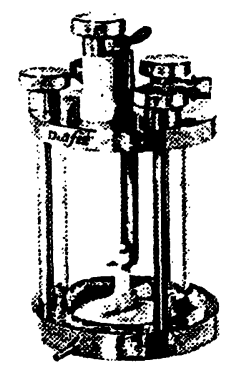

AMICON

ULTRAFILTRATIONS-

ZELLEN erlauben das

Arbeiten mit biologischen

Flüssigkeiten von

1 ML BIS ZU 20 LITERN

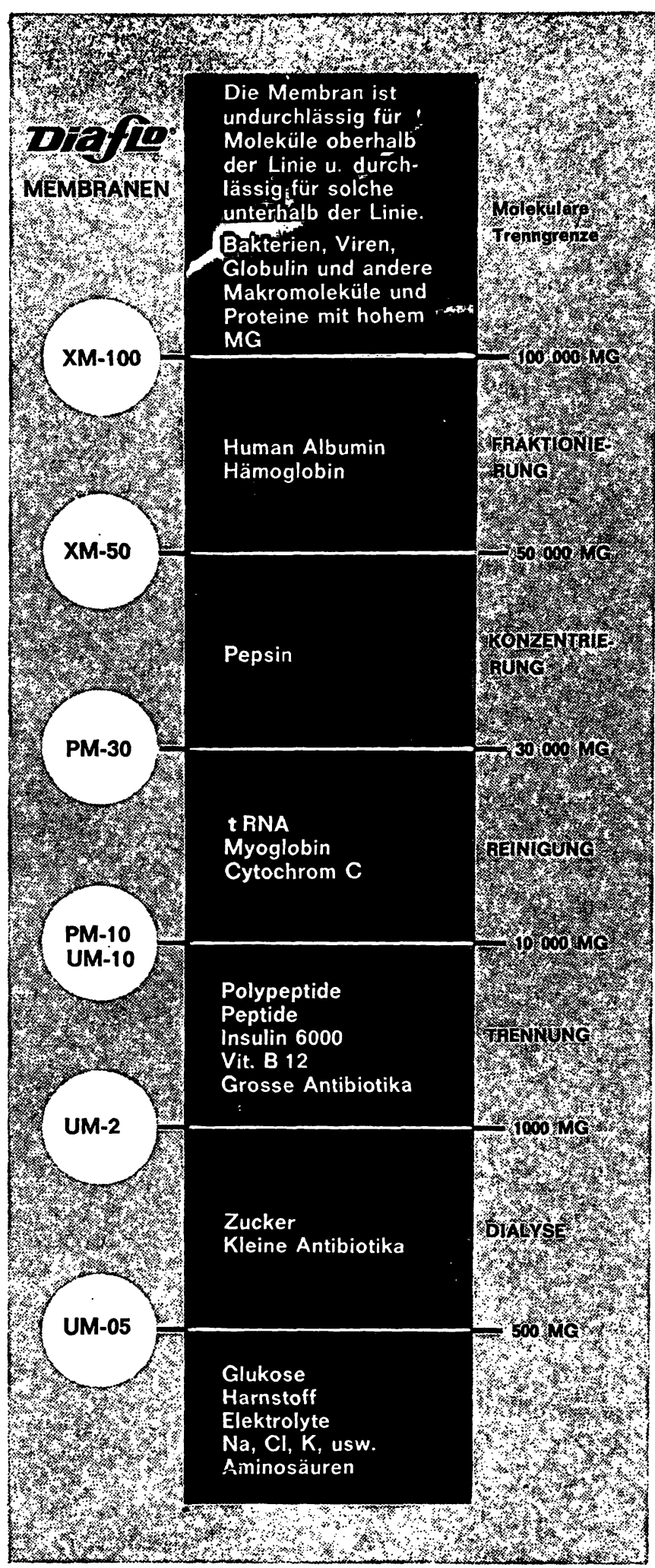

Amicon bietet ausserdem grössere industrielle Anlagen für kontinuierliche Verfahren.

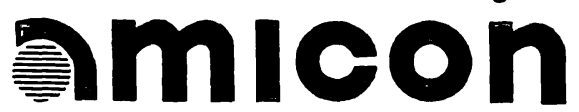

Abt.: 05

AMICON N.V. In den U.S.A.:

Heemskerckstraat 43 27 Hartwell Ave.
HOLLAND Ruf : (070) 642820 MASS. 02173 
ten $C_{4}$-Konzentrats im Bereich von $235-275 \mathrm{~nm}$ wieder (0,5 cm-Küvetten).

Der Magenextrakt war seinerzeit aus Unkenntnis des genauen Absorptionsverlaufs bei $268 \mathrm{~nm}$ nicht ausgemessen worden (unterbrochen gezeichneter Abschnitt in Abb. 12b). Eine Konzentrierung und Vorreinigung durch Extraktion wird für die UV-spektrometrische Erfassung der Benzalkoniumchloride am Organmaterial in der Regel deshalb erforderlich sein, weil der Absorptionsverlauf erst bei relativ hohen Konzentrationen deutlich ausgeprägt ist.

Das UV-Spektrum allein erlaubt im Falle des Benzalkoniumchlorids keine eindeutige Diagnose, da u. a. auch weitere benzylgruppenhaltige quartäre Ammoniumverbindungen, wie z. B. im Fesiamon (Hydramon), sehr ähnliche Absorptionskurven ergeben.

Eine einfache und rasche Differenzierungsmöglichkeit verschicdener quartärer Ammoniumverbindungen bei gleichzeitiger halbquantitativer Bestimmung mit Hilfe der Dünnschichtchromatographie sowie eine schnelle quantitative spektralanalytische Bestimmung kleinster Benzalkoniumchlorid-Konzentrationen (ab etwa $1 \mu \mathrm{g} / \mathrm{g}$ Material) nach Extraktion der Basen als Pikratkomplex wurden kürzlich beschrieben $(2,3)$.

Chlorochin (Resochin, Chloroquine, 7-Chlor-4,4'diäthylamino-1'-methylbutylamino-chinolindiphosphat) Akut-tödlicbe Vergiftung durcb Cblorocbin (Tgb.-Nr.: B 34/66)

Die Sektion erfolgte am 5. Tag p. m. Es wurden etwa $150 \mathrm{~g}$ Mageninhalt zur Untersuchung übergeben.

Abbildung 13 zeigt die UV-Spektren des Abdampfrückstandes eines Ätherextraktes aus der alkalisierten Mageninhalts-Probe (a) und einer Chlorochin-Testlösung (etwa $25 \mu \mathrm{g} / \mathrm{m} l$ ) (b) jeweils in 0,2N Schwefelsäure. Das Spektrum einer wäßr. neutralen ChlorochinLösung ist hiermit identisch $(0,5 \mathrm{~cm}$-Küvetten).

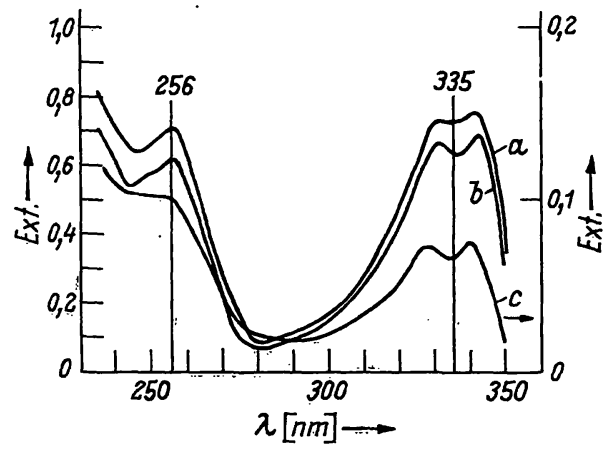

Abb. 13

Chlorochin-(Chloroquine-)Intoxikation, vgl. auch Text

Die Mageninhalts-Probe enthielt $z$. Z. des Todes noch mindestens $600 \mathrm{mg}$ an chemisch unveränderter Chlorochin-Wirksubstanz. An einer wäßr. Verdünnung des nach der Tabletteneinnahme Erbrochenen oder der Original-Mageninhalts-Probe wäre der eindeutige Nachweis wegen der Prägnanz der Absorptionskurve, besonders im langwelligen UV-Bereich, ebenfalls möglich gewesen, wie durch spätere Vergleichsuntersuchungen festgestellt wurde. Um die Aussagekraft spektralanalytischer Befunde zu demonstrieren, ist in einem anderen Maßstab (rechte Ordinate) die UV-Kurve eines Dünnschichtchromatogramm-Eluats von einem weiteren tödlichen Vergiftungsfall, einem vollendeten Suizid (Tgb.-Nr.: B 131/65) unter Mitwirkung von Chlorochin dargestellt, bei dem diese Substanz im Mageninhalt nur noch in Spuren neben größeren Mengen anderer toxischer Substanzen (Barbiturate) vorlag. (Eine ausführlichere Mitteilung über diese Vergiftungsfälle ist in Vorbercitung.)
Dormutil (Revonal, Methaqualon, 2-Methyl-3-o-tolyl4(3H)-chinazolinon)

Untersucbung an Erbrocbenem wegen Vergiftungsverdacbts mit unbekannten Mitteln (Tgb.-Nr.: B 128/64)

Am übersandten Erbrochenen waren makroskopisch Teile von Tabletten erkennbar.

Abbildung 14 gibt- die UV-Spektren der mechanisch abgetrennten erbrochenen Tablettenpartikel (a) und die von Dormutil-Tablettensubstanz (b) in Chloroform wieder. Zum Vergleich sind in dieser Abbildung außer-

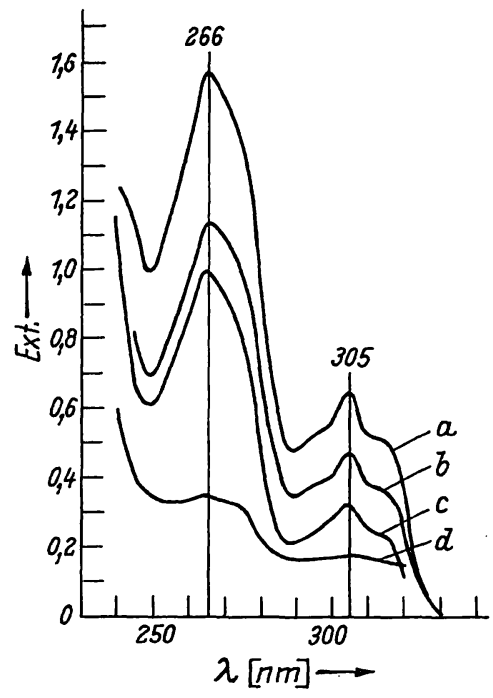

Abb. 14

Dormutil-(Methaqualon-)Intoxikation, vgl. auch Text

dem die Spektren einer in Wasser aufgenommenen, eingetrockneten Original-Mageninhalts-Probe (nach 21/2jähriger Lagerung) einer Wasserleiche (Tgb.-Nr.: B 1581 65) (d) und von Dormutil-Tablettensubstanz in Wasser (c) dargestellt. Die UV-Spektren von Methaqualon in Wasser und in Chloroform sind identisch $(0,5 \mathrm{~cm}$ Küvetten).

Ospolot (Sultiamum, N-(4'-Sulfamylphenyl-)butansultạm-(1,4))

Suizidale tödliche Ospolot-Vergiftung (Tgb.-Nr.: B 54/68)

In Abbildung 15 sind die Spektren wäßr. Lösungen von Ätherextraktionsrückständen der Leichen-MageninhaltsProbe (Spülflüssigkeitsreste) (a) und einer OspolotTablettensuspension (b) wiedergegeben. Zum Vergleich ist das Spektrum einer Ospolot-Tablettenlösung in Wasser (c) eingezeichnet (1 cm-Küvetten).

Der Verlauf der Kurve a entspricht dem abgetrennten Sultiamum aus $0,12 \mathrm{ml}$ Mageninhalt $/ \mathrm{m} l$ Meßlösung.

Die uns übergebene Mageninhalts-Probe $(150 \mathrm{~m} /$ trübe, schmutzig braune Flüssigkeit; Sektion 3 Tage p. m.) cnthielt ctwa $45 \mathrm{mg}$ Sultiamum. An einer Mageninhalts-Probe vor der Spülung wie auch an der ersten Spülflüssigkeit wäre die Erkennung des Wirkstoffes zweifellos direkt nach eincr Verdünnung mit Wasser möglich gewesen. (Ausführliche Mittcilung über die Analytik der Sultiamum-Intoxikation (4).) 


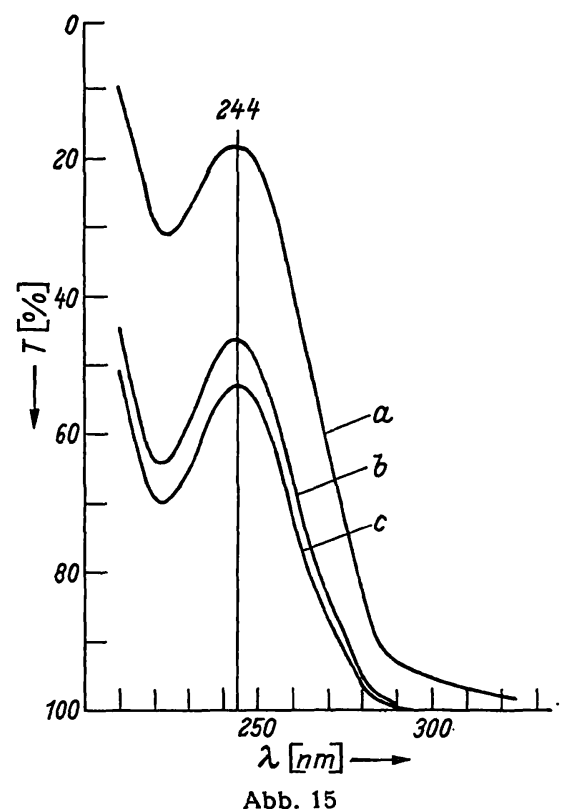

Ospolot-(Sultiamum-)Intoxikation, vgl. auch Text

\section{Nicotin}

Vollendeter Suizid mit dem Pflanzenscbutzmittel-Präparat "Nikotin 95/98\%" (Tgb.-Nr.: B 120/64)

Die Obduktion erfolgte am 3. Tag p. m.

Zur Untersuchung gelangten u. a. etwa $6 \mathrm{~m} /$ einer rötlichbraunen, alkalischen Mageninhalts-Probe.

In Abbildung 16 sind die UV-Spektren eines 1 : 2000 verdünnten Teils der Mageninhalts-Probe (b) - hier nach einjähriger Lagerung - sowie diejenigen der TatTrinkflüssigkeit ,Nikotin 95/98\%" (etwa $48 \mu \mathrm{g}$ Nicotin/ $\mathrm{ml}$ ) (a) und von Nicotin-reinst (etwa $25,5 \mu \mathrm{g} / \mathrm{ml}$ ) (c) im Bereich von 230 bis $290 \mathrm{~nm}$ dargestellt $(0,5 \mathrm{~cm}$ Küvetten).

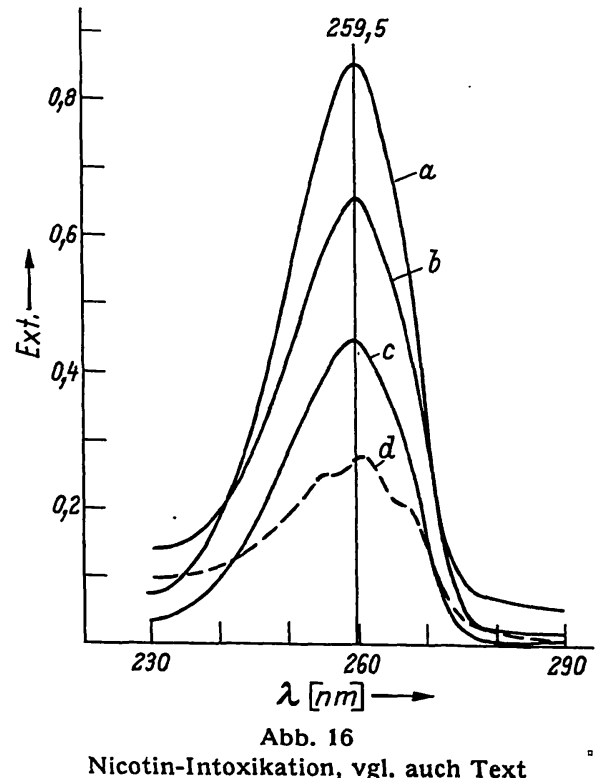

Die Verdünnungen erfolgten in diesem Fall mit $0,6 \mathrm{~N}_{3} \mathrm{PO}_{4}$, da die Mageninhalts-Probe wegen ihres Gehalts an Nicotin-Base alkalisch reagierte und UV-Spektren von Nicotin in verd. $\mathrm{H}_{3} \mathrm{PO}_{4}$ in der toxikologischen Literatur bekannt sind (siehe bei (5)). Die Art der verwendeten Mineralsäure ist jedoch ohne Bedeutung. Die Erkennung wäre auch an einer Verdünnung mit Wasser möglich gewesen, wie anhand des unterbrochen eingezeichneten Spek- trums von Nicotin-reinst in Wasser (d) demonstriert wird. Das Absorptionsmaximum zeigt in diesem Medium eine leichte bathochrome Verschiebung von etwa $259,5 \mathrm{~nm} \mathrm{zu} 261 \mathrm{~nm}$ und es ist von zwei typischen schulterförmigen Absorptionen umgeben (vgl. Pyridin-Spektrum).

Die Leichen-Mageninhalts-Probe enthielt etwa $66 \mathrm{mg} \mathrm{Nicotin} / \mathrm{m} /$. (Näheres zur Analytik dieser Vergiftung siehe unter (5).)

\section{Diskussion}

Während schnelle und sichere Ausmittlungen und Bestimmungen der für Suizidzwecke verbreiteten exogenen Vergiftungsmittel wie auch gezielt durchführbare Untersuchungen auf seltenere Wirkstoffe beim gegenwärtigen Stand der Analytik an geeigneten biologischen Materialien kaum problematisch sind (6), bereitet eine Suchanalyse unter Berückșichtigung aller möglichen Giftstoffe in der klinisch-chemischen wie auch in der forensisch-toxikologischen Analytik häufig erhebliche Schwierigkeiten.

In Anbetracht der gegenwärtigen und zukünftigen toxikologischen Gesamtsituation, insbesondere der verbreiteten Zugänglichkeit verschiedenartigster toxischrelevanter Mittel, des zunehmend hohen Anteils an Suizidversuchen durch Kombination unterschiedlicher Wirksubstanzen, der mangelhaften Erfaßbarkeit einer Reihe hochtoxischer Stoffe bei üblichen orientierenden Analysenverfahren sowie der allgemein geringen Spezifität klinischer und pathomorphologischer Vergiftungssymptome, erscheinen einfache, zeit- und materialsparende Verfahren, die - wie das hier mitgeteilte in einem Arbeitsgang ohne Materialzerstörung die Erfassung und die Klassifizierung des überwiegenden Teils der verbreiteten und der seltenen organischen Vergiftungsmittel und ggf. gleichzeitig deren semiquantitative Bestimmung ermöglichen, vor allem für die klinisch-toxikologische Untersuchungstätigkeit von Interesse.

Die UV-Spektrometrie gewinnt seit ihrer Einführung in die chemische Analytik auch für die toxikologische. Chemie zunehmend an Bedeutung. Trotz der Vielzahl beschriebener UV-spektrometrischer Untersuchungsmethoden sind ihre Einsatzmöglichkeiten bei weitem noch nicht ausgeschöpft, wie in dieser Mitteilung gezeigt werden konnte. Eine zusammenhängende Darstellung zur anstehenden Thematik liegt $u$. W. in der Literatur nicht vor.

Eine eingehendere Besprechung der UV-spektrometrischen Analytik im allgemeinen - hierüber existieren bereits zahlreiche Publikationen (Lit. siehe bei $(7,8)$ ) wie auch der ausgewählten Vergifțungsfälle ist im Rahmen dieser Mitteilung nicht möglich. Die weitere Diskussion muß deshalb auf einige für die praktische Anwendung der Verfahrensweise bedeutsame Punkte beschränkt werden.

Die im Vergleich zum IR-Spektrum geringe Informationșkapazität eines UV-Spektrums darf in der analytischen Toxikologie nur sehr bedingt als ein Mangel angesehen werden. Die UV-spektrometrische Analytik stellt relativ geringe Anforderungen an den Analytiker und an die chemische Reinheit der zu untersuchenden 
Lösungen. Die Unkompliziertheit der UV-Spektren macht diese schon deshalb zu einem wertvollen Hilfsmittel, weil es bei Suchanalysen in erster Linie darauf ankommt, schnell das Vorliegen von UV-absorbierenden Fremdstoffen auszuschließen oder zu erkennen und diese ggf. zu klassifizieren. Der Informationswert von UV-Spektren steigt in dem $\mathrm{Maße}$, wie die gesetzmäßigen chemisch-physikalischen Beziehungen zwischen Absorption und Konstitution der Substanzen, die Beschaffenheit der zu untersuchenden Proben, insbesondere die Einflüsse des Lösungsmittels und die pH-Werte der Lösungen, sowie die evtl. vorangegangenen Bearbeitungsbedingungen (Aufbereitung, Anreichetung, Reinigung und Trennung) bei ihrer Bewertung berücksichtigt werden.

Alle Mageninhalts-Proben sollten unmittelbar nach der Gewinnung oder dem Eingang, spätestens aber im $\mathrm{Zu}-$ sammenhang mit der UV-spektrometrischen Untersuchung makroskopisch hinsichtlich Farbe, Konsistenz und Bestandteile sowie bezüglich ihres $\mathrm{pH}$-Wertes und Geruchs geprüft werden. Bei geruchlicher Auffälligkeit empfehlen sich gezielte Tests mittels Gasspürröhrchen (s. bei (9)). Enthalten die Proben Fremdfarbstoffe, so kann die zusätzliche Aufnahme von Spektren im sichtbaren Bereich (vgl. VIS-Spektren in Abb. 9, II, Drageefarbstoff) nützlich sein.

Sofern es sich nicht ausschließlich um eine Bestätigungsanalyse handelt, sind zur Befundsicherung Parallel- oder Folgeuntersuchungen mittels Analysenverfahren durchzuführen, die von der UV-Spektrometrie unabhängig sind. Als in diesem Sinne für eine allgemeine Anwendung besonders geeignet ist die Dünnschichtchromatographie $(10,11,12)$ anzusehen die unter standardisierten Bedingungen und bei zweckmäßiger Kombination mit der UV-Spektrometrie $(13,14,15,16)$ zur schnellen Identifizierung von über $95 \%$ der wenig flüchtigen organischen Vergiftungsmittel führen kann. Bei akuten oralen Vergiftungen vermag diese Methode auch durch Aufbringen unbearbeiteter Mageninhalts-Proben gute Ergebnisse zu liefern (16).

Die im Mageninhalt (1. Giftweg bei oraler Applikation) nachgewiesenen, nicht resorbierten Fremdstoffe müssen nicht in jedem Fall zwangsläufig mit den Hauptwirk- stoffen im 2. Giftweg (Wirksphäre) identisch sein. Deshalb sind die analytischen Untersuchungen nach Möglichkeit - in forensischen Fällen stets - auf Proben des 2. und/oder 3. Giftweges auszudehnen, bevor eine endgültige, analytisch fundierte toxikologische Diagnose erstattet wird. Die analytische Untersuchung und die Befundbewertung werden sehr gefördert, wenn der Analytiker über alle diesbezüglich wesentlichen Ergebnisse der Ermittlung und der Klinik auf dem laufenden gehalten wird. Schon die Kenntnis des wahrscheinlichen Zeitraums zwischen Gifteinnahme oder Auffindung des Betroffenen und der Gewinnung der Proben ist ein bedeutsamer Faktor zur Beantwortung der Frage, ob und inwieweit z. B. allein der UV-spektrometrische Mageninhalts-Befund zur Erklärung der Vergiftungssymptomatik herangezogen werden kann. Die kritiklose Bewertung einer einzelnen UV-spektrometrischen Untersuchung oder gar nur der ausgemessenen Maxima und Minima kann zu schwerwiegenden Fehlaussagen führen (17).

Die oftmals umstrittene Frage nach der Indikation und dem Erfolg einer Magenspülung (siehe u. a. $(18,19)$ ) läßt mit Hilfe der beschriebenen Verfahrensweise in vielen Fällen eine schnelle und eindeutige Klärung zu, da für ihre Durchführung - je nach Beschaffenheit der Proben, der Anzahl notwendiger Kontrollen und der Leistungsfähigkeit des Spektralphotometers - allgemein nur 20 bis $60 \mathrm{Min}$. benötigt werden.

Einrichtungen, die nicht über ausreichende Erfahrungen in der UV-Spektrometrie und geeignete Spektrensammlungen verfügen, sollten zwecks Bewertung unbekannter Spektren wie auch in Zweifelsfällen Informationsund Dokumentationsstellen für toxikologisch-analytische Daten zu Rate ziehen. An die Industrie und die Zulassungskommissionen ergibt sich die Forderung, diesen Stellen künftig nach einheitlichen Grundbedingungen gewonnene spektralanalytische Daten von allen toxisch-relevanten Mitteln bekannt zu geben, bevor solche Mittel einem größeren Personenkreis zugänglich gemacht werden.

Für die stets zuverlässige Hilfe bei der Durchführung der langjährigen Untersuchungen sei insbesondere der ltd. techn. Assistentị Fräulein H. BREMER auch an dieser Stelle gedankt.

\section{Literatur}

1. Dunlop, M. und D. H. Curnow, J. Clin. Path. London 20, 204 (1967). - 2. Tress, D. und K.-H. NAGEL, Arch. Toxikol. 22, 333 (1967). - 3. TrEss, D. und K. Rörzscher, Atch. Toxikol. 25, 183 (1969). - 4. Ahrend, K.-F., L. Nagy und D. Tress, Arch. Toxikol. 25, 229 (1969). - 5. Tiess, D. und K.-H. NAgeL, Arch. Toxikol. 22, 68 (1966). - 6. Cilarke, E. G. C., Isolation and identification of drugs, Pharmaceutical Press, London (1969). 7. Derkosch, J., Absorptionsspektralanalyse im ultravioletten, sichtbaren und infraroten Gebiet, Akad. Verl. Gesellsch. Geest und Portig, Leipzig (1967). - 8. Neugebauer, F. A., in: GadaMERs Lehrbuch der chemischen Toxikologie und Anleitung zur Ausmittlung der Gifte, II, 3. Aufl., S. $426 \mathrm{ff}$., Vandenhocck u. Ruprecht, Göttingen (1966). - 9. KLöckING, H. P., Zschr. ärztl. Fortbild. 61, 918 (1967). - 10. STAkr, E., Dünnschicht-Chroma- tographie, 2. Aufl., Springer Verlag, Berlin (1967). - 11. MACHATA, G., Dtsch. Zschr. gerichtl. Med. 59, 181 (1967). - 12. WALDr, D., in: Gadamers Lehrbuch der chemischen Toxikologie und Anleitung zur Ausmittlung der Gifte, II, 3. Aufl., S. 83 ff., Vandenhoeck u. Ruprecht, Göttingen (1966). - 13. KISSER, W. und G. Machata, Mikrochim. Acta 1968, 374. - 14. Ahrend, K.-F. und D. Tress, Verh. Dtsch. Ges. exper. Med. (im Druck). 15. Schмпd, GG., in: Gerichtliche Medizin und Kriminalistik, Festschrift für E. WeINIG, Verlag Georg Schmidt-Römhild, Lübeck (1964). - 16. Gotrschald, S. und D. Tress, Verh. Dtsch. Ges. exper. Med. (im Druck). - 17. GoldnAum, L. R., E. H. Johnston und J. M. BlumberG, J. Forensic. Sci. 8, 286 (1963). 18. Ibe, K., Med. Klin. 46, 1832 (1966). - 19. Balzerert, F. und W. Arnold, Dtsch. med. Wschr. 21, 485 (1966). 